\title{
Mechanism of Signal Amplification in the Olfactory Sensory Cilia
}

\author{
Hiroko Takeuchi and Takashi Kurahashi \\ Department of Frontier Biosciences, Osaka University, Toyonaka, Osaka 560-8531, Japan
}

\begin{abstract}
Molecular mechanisms underlying olfactory signal amplification were investigated by monitoring cAMP dynamics in the intact sensory cilia. We saw that $[\mathrm{cAMP}]_{\mathrm{i}}$ increased superlinearly with time during odorant stimuli for $>1 \mathrm{~s}$. This time course was remarkably different from that obtained with the rapid quench method previously applied to the in vitro preparation, in which $[\mathrm{cAMP}]_{\mathrm{i}}$ change has been reported to be transient. The superlinear increase of $[\mathrm{cAMP}]_{\mathrm{i}}$ was attributable to a gradual increase of cAMP production rate that was consistent with the thermodynamical interaction model between elemental molecules, as has been revealed on the rod photoreceptor cell. It thus seems likely that the fundamental mechanism for molecular interactions between olfactory transduction elements is similar to that of the rod. In olfaction, however, cAMP production was extremely small ( $\sim 200,000$ molecules/s/cell at the maximum), in contrast to the cGMP hydrolysis in the rod (250,000 molecules/photon). The observed numbers indicate that the olfactory receptor cell has lower amplification at the enzymatic cascade. Seemingly, such low amplification is a disadvantage for the signal transduction, but this unique mechanism would be essential to reduce the loss of ATP that is broadly used for the activities of cells. Apparently, transduction by a smaller number of second-messenger formations would be achieved by the fine ciliary structure that has a high surface-volume ratio. In addition, it is speculated that this low amplification at their enzymatic processes may be the reason why the olfactory receptor cell has acquired high amplification at the final stage of transduction channels, using $\mathrm{Ca}^{2+}$ as a third messenger.
\end{abstract}

Key words: olfaction; rod; cAMP; adenylyl cyclase; ion channel; signal transduction

\section{Introduction}

In the olfactory sensory cilia, odorant binding to the G-proteincoupled receptor (GPCR) (Buck and Axel, 1991) triggers adenylyl cyclase (AC)-cAMP system (Pace et al., 1985; Sklar et al., 1986; Jones and Reed, 1989; Lowe et al., 1989; Bakalyar and Reed, 1990), which leads to the openings of ion channels that underlie cell excitation (Kurahashi, 1989; Firestein et al., 1990; Lowe and Gold, 1991). Although the natural ligands for olfaction have large diversity exceeding 100,000 varieties, the signal thus converges into only one second messenger, cAMP, through the enzymatic cascade equipped in the sensory cilia (Takeuchi and Kurahashi, 2003; Takeuchi et al., 2003).

Generally, signal amplification is essential for the sensory transduction as has been exemplified in the case of the rod photoreceptor cell in which only one photon triggers cGMP change by 250,000 molecules. In case of olfaction, however, the information about signal amplification is completely lacked, especially in

\footnotetext{
Received March 27, 2005; revised 0ct. 12, 2005; accepted 0ct. 12, 2005.

This work was supported by grants from the Human Frontier Science Program (T.K.), Ministry of Environment (T.K.), the Research Foundation for Opto-Science and Technology (T.K.), Ministry of Education, Culture, Sports, Science, and Technology (H.T., T.K.), and the Kao Foundation for Arts and Sciences (H.T.). We thank Drs. Akimich Kaneko, Trevor D. Lamb, and Edward N. Pugh Jr for their comments on the early stage of this work. We also thank Dr. Heinz Breer and members in his laboratory for discussion and Drs. Anna Bocaccio, Anna Menini, Vincent Torre, and members of Scuola Internazionale Superiore di Studi Advanzati for their comments on the final version of this manuscript.

Correspondence should be addressed to Hiroko Takeuchi, Department of Frontier Biosciences, Osaka University, Toyonaka, 0saka 560-8531, Japan. E-mail: hiroko@bpe.es.osaka-u.ac.jp.

DOI:10.1523/JNEUROSCI.1931-05.2005

Copyright $\odot 2005$ Society for Neuroscience $\quad$ 0270-6474/05/2511084-08\$15.00/0
}

its enzymatic cascade. This is mainly attributable to the technical difficulties accompanied with the nano-scale structure of sensory cilia. In addition, olfactory cilia are heterogeneous in terms of their responsiveness to varieties of odorant species. Such heterogeneous responsiveness makes the quantitative analysis for cAMP production (e.g., measurement for absolute $[\mathrm{cAMP}]_{\mathrm{i}}$ ) quite difficult with the biochemical assay. If one applies a certain odorant to the crude material, it is difficult to know how much fraction of preparations responds to the applied species of odorant.

In the present study, $[\mathrm{cAMP}]_{\mathrm{i}}$, cytoplasmic cAMP dynamics, and $\mathrm{AC}$ activities were investigated during the odorant response in living olfactory receptor cells. By using single-cell preparations, we could screen out the sample that responded to the particular odorant, regardless of the heterogeneity of the olfactory sensory system. To overcome the difficulty of monitoring the molecular dynamics within nano-scale sensory cilia, we used a combined technique of patch-clamp and photo-activated compounds. First, the activities of cyclic nucleotide-induced membrane currents were monitored, whereas $[\mathrm{cAMP}]_{\mathrm{i}}$ was freely manipulated through the UV-photolysis of intracellulary loaded caged compounds. Because in this condition the production of cAMP is proportional to the intensity of light, we could obtain a calibration for cytoplasmic $[\mathrm{cAMP}]_{\mathrm{i}}$ as a function of the membrane current. Based on the current- $[\mathrm{cAMP}]_{\mathrm{i}}$ relationship, we then monitored ligand (odorant)-induced $[\mathrm{cAMP}]_{\mathrm{i}}$ change and $A C$ activities within the cilia. It was shown that the $[\mathrm{CAMP}]_{\mathrm{i}}$ increased superlinearly with time for $>1 \mathrm{~s}$, which was a result of a 
gradual increase of cAMP production rate. Interestingly, a linear increase of effecter enzyme activities is theoretically consistent with the model describing the G-protein-mediated signaling cascade of the rod photoreceptor cell (Pugh and Lamb, 1990; Lamb and Pugh, 1992). In this aspect, it is assumed that molecular interactions in both systems resemble each other. However, the number of the cAMP molecules produced in the olfactory receptor cell was very small in comparison with that of the cGMP hydrolysis in the rod. Furthermore, it was found that the cAMP concentration range was almost equivalent to that of the effective dose-range of odorant as a native ligand. These results suggest that the olfactory signal transduction system has a low molar amplification system up to the production of cAMP. It is reasonably considered, however, that such low amplification system has been established to achieve an efficient energy conversion to reduce the loss of ATP that is broadly used for the activities of cells. Instead, transduction by a smaller number of second-messenger formations would be achieved by the fine ciliary structure that has a high surface-volume ratio. In addition, it is speculated that the olfactory receptor cell might have acquired high amplification at the final stage of transduction, namely, signal boosting by ion channels using $\mathrm{Ca}^{2+}$ as a third messenger.

\section{Materials and Methods}

Recordings and odor stimulation. Single receptor cells were enzymatically ( $0.1 \%$ collagenase) dissociated from the olfactory epithelium of the newt Cynops pyrrhogaster, as described previously (Kurahashi, 1989; Takeuchi and Kurahashi, 2002). Cells were bathed in the normal Ringer's solution containing the following (in $\mathrm{mm}$ ): $110 \mathrm{NaCl}, 3.7 \mathrm{KCl}, 3 \mathrm{CaCl}_{2}, 1 \mathrm{MgCl}_{2}$, 10 HEPES, 15 glucose, and 1 pyrvate, $\mathrm{pH}$ adjusted to 7.4 with $\mathrm{NaOH}$, before use. Experiments were performed at room temperature $\left(23-25^{\circ} \mathrm{C}\right)$.

Membrane currents were recorded with the whole-cell recording configuration (Hamill et al., 1981). Patch pipettes were made of borosilicate tubing with filament (outer diameter, $1.5 \mathrm{~mm}$; World Precision Instruments, Berlin, Germany) by using a two-stage vertical patch electrode puller (PP-830; Narishige Scientific Instruments, Tokyo, Japan). The recording pipette was filled with a solution containing $119 \mathrm{~mm} \mathrm{CsCl}$ (plus $1 \mathrm{mM} \mathrm{CaCl}_{2} ; 5$ mM EGTA, pH was adjusted to 7.4 with HEPES buffer) to suppress $\mathrm{K}^{+}$channels, which cause large current fluctuations. The pipette resistance was $10-15 \mathrm{M} \Omega$. Normal Ringer's solution (see above for composition) or $\mathrm{Ca}^{2+}$-free solution (the solution to which no $\mathrm{Ca}^{2+}$ was added) was used as the external solution. In the present study, we did not use any $\mathrm{Ca}^{2+}$ buffer in the external solution, because in the physiological experiments for solitary olfactory cells, it has been widely believed that low-Ca ${ }^{2+}$ condition makes the conditions of cells extremely unstable. $\mathrm{Ca}^{2+}$ activity in the present experimental condition has been thought to be $\sim 10 \mu \mathrm{M}$ (Kurahashi, 1990).

The recording pipette was connected to a patch-clamp amplifier (Axopatch 1D; Molecular Devices, Foster City, CA). The signal was low-pass filtered at $0.5 \mathrm{kHz}$, digitized by an $\mathrm{A} / \mathrm{D}$ converter (sampling frequency, 1 $\mathrm{kHz}$ ), and connected to a single-task computer (operated using MSDOS; Microsoft, Redmond, WA) (PC9821, 80486CPU; NEC, Tokyo, Japan). Simultaneously, signals were monitored on an oscilloscope and recorded on a chart recorder. Light and odor stimuli and the data acquisition were regulated by the same computer using an original program. The results were analyzed by an off-line computer and plotted by using Microcal Origin 7.5 software (OriginLab, Northampton, MA). For curve drawings and analyses, data were smoothened by $50 \mathrm{~Hz}$ averaging, or data sampled at $1 / 16 \mathrm{kHz}$ were used. This smoothing procedure was a crucial step to obtain stable results, especially for taking the derivative of waves.

For odorant stimuli, cineole (1 mm) (eukalyptos; Katayama Chemical, Osaka, Japan) was dissolved in the external solution. The stimulus solution was put into a puffer pipette and was applied to the cell under the recording by using a pressure ejection system, which was controlled by a single-task computer (operated using MS-DOS) (Ito et al., 1995). The actual concentration of the odorant that stimulated the cell was therefore dependent on the pressure applied to the puffer pipette. We had confirmed that the pressure range used in the present work did not reach to the saturation (therefore, lower than $1 \mathrm{~mm}$ ). The timing of the pressure pulses was shown above the current traces in the figures.

Photolysis of caged cyclic nucleotides. The olfactory receptor cell was used throughout the experiments (beginning from the $[\mathrm{cAMP}]_{\mathrm{i}}$ calibration) to keep loadings and photolysis of caged cAMP (adenosine $3^{\prime} 5^{\prime}$ cyclic monophosphate, P1-(2-nitrophenyl)ethyl ester; Calbiochem, La Jolla, CA) identical. We recognized that the efficiency for the caged cAMP loading or photolysis is varied between preparations (see larger SD bars at strong intensities in Fig. $4 D$ ). Therefore, parameters used for calculation were obtained statistically. Caged cAMP was dissolved in 100 $\mathrm{mm}$ dimethylsulphozide and stored frozen at $-20^{\circ} \mathrm{C}$ in complete darkness. The stock solution was diluted into Cs-pipette solution to a final concentration of $1 \mathrm{~mm}$ before each experiment. Free cyclic nucleotide contamination within the commercial-available caged compound was estimated to be $2 \%$ when measured with caged cGMP compound [P1(2-nitrophenyl) ethyl ester; Calbiochem] by using the rod outer segment membrane as a cGMP probe. This value indicates that basal-free cyclic nucleotide monophosphate (cNMP) does not affect cyclic nucleotidegated $(\mathrm{CNG})$ channel in the ciliary membrane, because $2 \%$ contamination $(20 \mu \mathrm{M})$ was smaller than the detection threshold [Kurahashi (1990), his Fig. 3]. Through the present experiment, in fact, we did not observe any sign for cAMP-induced response when the caged compound was introduced simultaneously with the establishment of the whole-cell recording configuration. It is highly likely that free cNMP is hydrolyzed during the diffusion along the dendritic lumen, most likely resulting from the endogenous phosphodiesterase (PDE) activities.

A UV light with a wide wavelength was applied to cover the ciliary region of the solitary cells through an epifluorescent system. A $100 \mathrm{~W}$ xenon lamp was used to induce photolysis. The timing and duration of light illumination were controlled by a magnetic shutter, and the light intensity was specified by a wedge filter under computer control. The light source provided the constant intensity of UV component while the magnetic shutter was opened (confirmed with a UV-sensitive photodiode). The light source was mechanically isolated from the inverted microscope to avoid transmission of vibrations. The timing of the UV flashes is shown above the current traces in the figures. The light intensities indicated here are relative values in reference to the maximum intensities being 1.0. Before all experiments, we applied a series of different intensities of light to confirm the maximum amplitude $\left(I_{\max }\right)$ and dynamic range of each cell. Especially, we paid a particular attention to avoid system saturation.

\section{Results}

Activities of odor-activated channels and cAMP-activated channels in the olfactory receptor cilia under physiological condition

Single olfactory receptor cells isolated from the newt were recorded with the whole-cell voltage-clamp technique, while cells were stimulated either by the odorant as a native ligand $(1 \mathrm{~mm}$ cineole in the puffer pipette) or by the UV light for photolysis of cytoplasmic caged cAMP (Fig. 1A). When cells were stimulated by cineole, 16 of 54 cells $(29.6 \%)$ showed inward current responses at $-50 \mathrm{mV}$ (Fig. $1 \mathrm{~B}$ ). The amplitude of the inward current was dose dependent, and the relationship was fitted by the Hill equation:

$$
I=I_{\max } \times \frac{C^{n_{H}}}{C^{n_{H}}+K_{1 / 2}^{n_{H}}},
$$

where $I$ is the current, $C$ is the dose of the ligand (or the intensity of light in photolysis experiments shown below), $K_{1 / 2}$ is the halfmaximum dose, and $n_{H}$ is the Hill coefficient, which was $6.7 \pm$ 1.9 (mean $\pm \mathrm{SD} ; n=5$ ) when measured in the present study (Fig. $1 C)$. Critically, however, this number may slightly be an overes- 
timation, because in our study, the odorant dose was regulated by changes in the pressure applied to the puffer pipette as has been used frequently in the experiments on single olfactory receptor cells. It has been suggested that changing pressure causes the change in the concentration and stimulus period simultaneously (especially at brief pulses). Therefore, the actual dose expresses a superlinear function against the pressure. However, even if we take this nonlinearity into account, the extremely high $n_{H}$ value would lead to a conclusion that the olfactory transduction system expresses very high cooperativities, as has been reported previously (Lowe and Gold, 1993a,b; Kleene, 1997; Takeuchi and Kurahashi, 2002, 2003; Matthews and Reisert, 2003).

Similarly, cytoplasmic photolysis of caged cAMP induced inward current responses with similar profiles as the odorant-induced current (Fig. $1 D, E)\left(n_{H}\right.$ $=5.1 \pm 1.9 ; n=21$ ) (Takeuchi and Kurahashi, 2002). This indicates that the nonlinear amplification is established at the downstream point from the cAMP production and has been interpreted to be attributed to the involvements of two different transduction channels, namely, CNG channels and $\mathrm{Ca}^{2+}$-activated $\mathrm{Cl}^{-}$channels (Fig. 1A). CNG channels express cooperativities of 1.2-1.6 (Nakamura and Gold, 1987; Kurahashi and Kaneko, 1991, 1993; Balasubramanian et al., 1996), and $\mathrm{Ca}^{2+}$-activated $\mathrm{Cl}^{-}$channels show cooperativities of $\sim 2$ (Kleene and Gesteland, 1991). Coupling both, the total current shows a very high cooperativity expressing nonlinear amplification.

\section{Relationship between UV intensity, $[\mathrm{cAMP}]_{\mathrm{i}}$, and membrane current}

To derive the cytoplasmic cAMP concentration $\left([\mathrm{cAMP}]_{\mathrm{i}}\right)$ from the membrane current, it was necessary to calibrate the relationship between $[\mathrm{cAMP}]_{\mathrm{i}}$ and olfactory CNG channels. In previous works, this relationship has already been investigated precisely for inside-out membrane patch preparations obtained from the olfactory cilia/knob (Nakamura and Gold, 1987; Kurahashi and Kaneko, 1991, 1993; Balasubramanian et al., 1996). In the whole-cell preparation, however, this relationship is unknown, because there was no way to control $[\mathrm{cAMP}]_{\mathrm{i}}$ precisely in living cilia. To resolve this technical difficulty, we used the photolysis of caged cAMP for manipulating cytoplasmic cAMP and made a direct comparison with dose dependence of the CNG channel, which has been described previously with the inside-out patch preparation.

Because in the previous inside-out studies experiments were performed under $\mathrm{Ca}^{2+}$-free condition, the observed current was carried through pure CNG channels and was not boosted by the $24.5 \mathrm{pA} ; K_{1 / 2}, 0.23$.

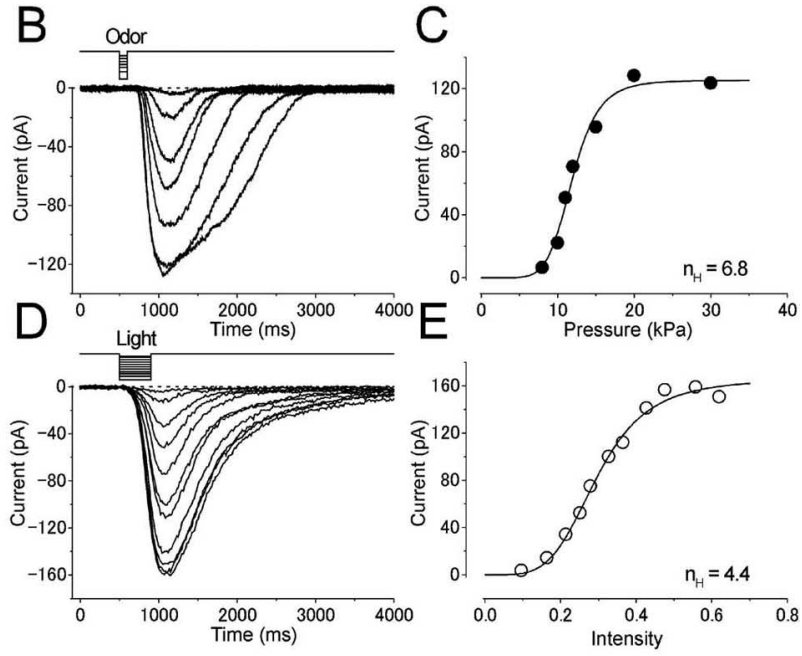

Figure 1. Intensity dependences of the odor- and light-induced responses. $A$, Experimental logic. Experiments were conducted to drive the native transduction pathway under a physiological condition. $V_{\mathrm{h}}=-50 \mathrm{mV}$. Odorant or light stimulation was applied to single olfactory receptor cells. $\boldsymbol{B}$, Membrane currents were recorded from a cell stimulated by odorant. The odorant dose was varied by changing the air pressure applied to the puffer pipette $(8,10,11,12,15,20$, and $30 \mathrm{kPa})$ while the duration was kept constant $(100 \mathrm{~ms})$. Downward deflections of the top trace indicate the timing and duration of the stimulation (throughout the figures). C, Dose-response relationship of the odorant-induced current. Peak amplitudes of responses obtained in $\boldsymbol{B}$ were plotted against the dose of odor. The smooth line was drawn by a least-square fitting of the data points by the Hill equation (Eq. 1). The Hil coefficient $\left(n_{H}\right)$ was 6.8 . $I_{\text {max }}, 125.3 \mathrm{pA} ; K_{1 / 2}, 11.9 \mathrm{kPa}$. $D$, Membrane currents were recorded from a cell loaded with $1 \mathrm{~mm}$ caged CAMP (different cell from $\boldsymbol{B}$ and $\boldsymbol{C}$ ). Light intensity was varied $(0.1,0.16,0.21,0.25,0.28,0.33,0.36,0.43,0.48,0.56$, and 0.62 ) ile the duration was kept constant $(500 \mathrm{~ms})$. $\boldsymbol{E}$, Intensity-response relationship of the light-induced current. Peak amplitudes of responses obtained in $\boldsymbol{D}$ were plotted against the intensity of light. The smooth line was drawn by a least-square fitting of the data points by the Hill equation. $n_{H}=4.4 . I_{\text {max }}, 165.8 \mathrm{pA} ; K_{1 / 2}, 0.3$.

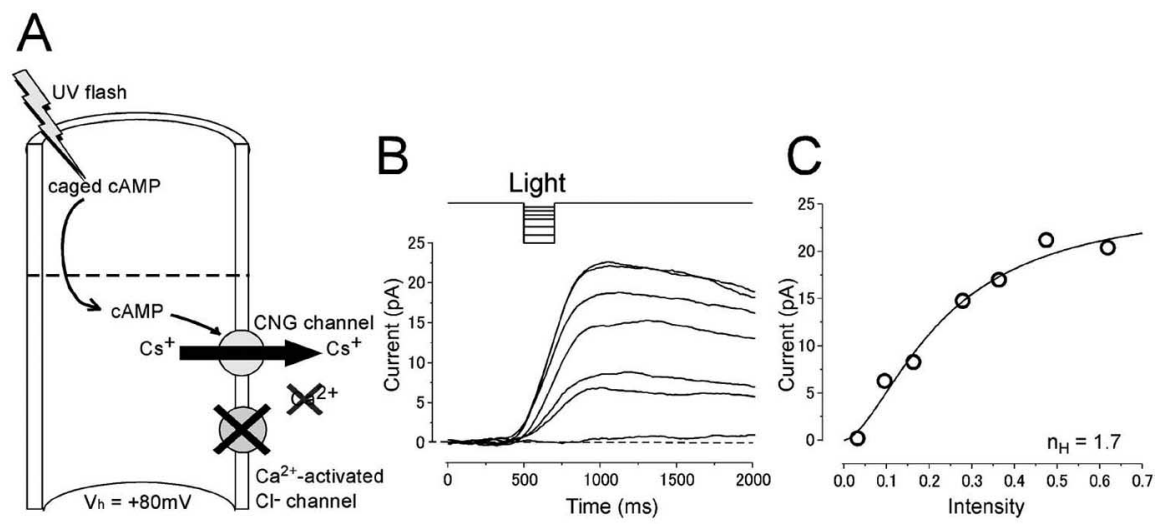

Figure 2. Current responses induced by light stimulation under the $\mathrm{Ca}^{2+}$-free condition. $\boldsymbol{A}$, Experimental logic. [CAMP $]_{\mathrm{i}}$ was jumped by a photolysis of caged cAMP in the absence of $\mathrm{Ca}^{2+}$ influx. $V_{\mathrm{h}}=+80 \mathrm{mV}$. $\boldsymbol{B}$, Light-induced responses. Light intensity was varied $(0.034,0.096,0.16,0.28,0.37,0.48$, and 0.62$)$ while the duration was kept constant $(200 \mathrm{~ms})$. $\boldsymbol{C}$, Intensity-response relationship of the light-induced current. Peak amplitudes of the responses obtained in $\boldsymbol{B}$ were plotted against the intensity of light. The solid line was drawn by the least-square fitting of the data points by the Hill equation (Eq. 1). $n_{\mathrm{H}^{\prime}} 1.7 ; I_{\max }$

effect of $\mathrm{Ca}^{2+}$-activated $\mathrm{Cl}^{-}$channels. To obtain absolute $[\mathrm{cAMP}]_{\mathrm{i}}$ in the present whole-cell recording condition, experiments were performed in a similar condition to which no $\mathrm{Ca}^{2+}$ was added $\left[\mathrm{Ca}^{2+}\right.$-free solution, having the same condition as in the study by Kurahashi and Kaneko (1991)]. Furthermore, the membrane was held at positive potentials $(+50$ to $+80 \mathrm{mV})$ to prevent the influx of $\mathrm{Ca}^{2+}$ into the ciliary cytoplasm (Fig. $2 \mathrm{~A}$ ). This experimental manipulation also removed other $\mathrm{Ca}^{2+}$ triggered effects equipped in the olfactory cell; one example has been known as the olfactory adaptation expressed by a $\mathrm{Ca}^{2+}$ feedback to the CNG channel (Chen and Yau, 1994; Kurahashi 

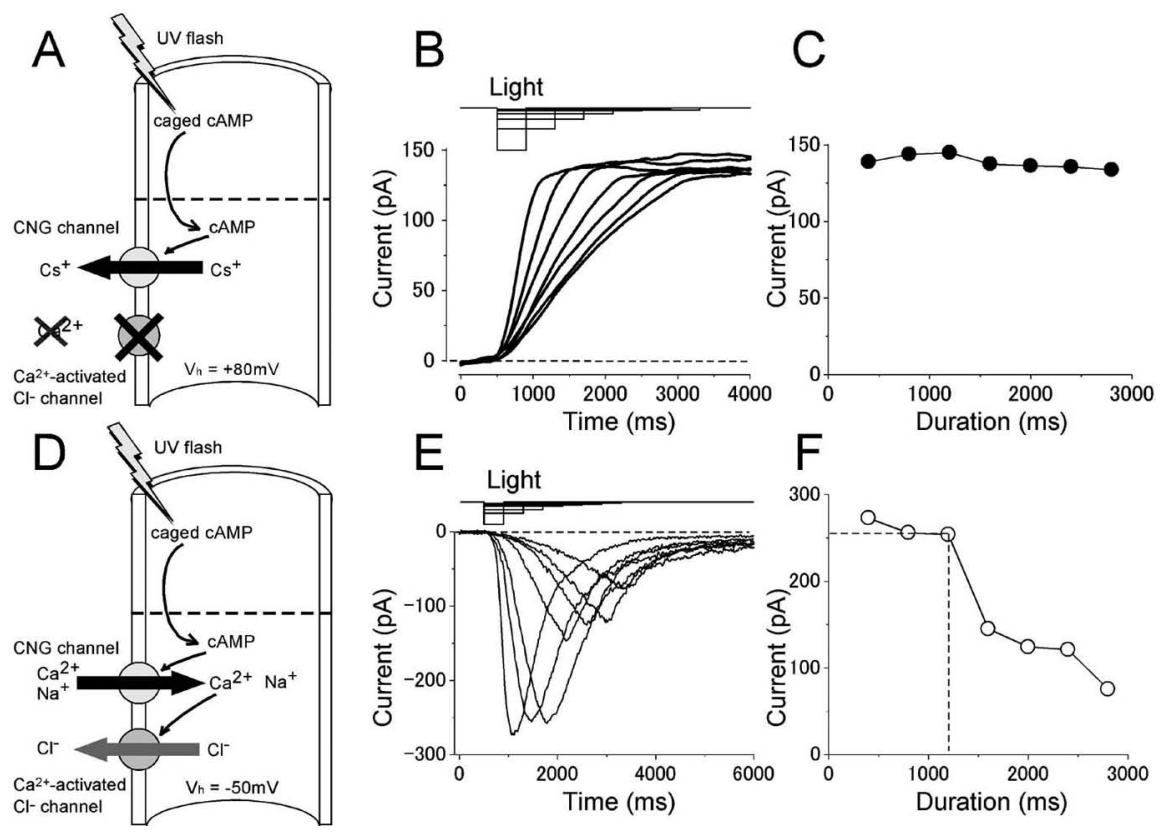

Figure 3. Reciprocity between the intensity and duration of the light under the absence and presence of $\mathrm{Ca}^{2+} . \boldsymbol{A}$, Experimental logic. [CAMP] ${ }_{i}$ was jumped by a photolysis of caged cAMP under the $\mathrm{Ca}^{2+}$-free condition. $V_{\mathrm{h}}=+80 \mathrm{mV}$. $\boldsymbol{B}$, Time dependence of the light-induced current under the $\mathrm{Ca}^{2+}$-free condition. Durations were 400,800,1200, 1600, 2000,2400, and $2800 \mathrm{~ms}$, and intensities were $0.62,0.31,0.21,0.15,0.12,0.10$, and 0.08 , respectively. The amplitude was confirmed to be a subsaturating level of this cell $\left(I_{\max }=171.1 \mathrm{pA}\right)$.C C Current amplitudes in $A$ were plotted against the duration of the light. Light induced current under the physiological condition. $\boldsymbol{D}$, Experimental logic. Experiments were conducted to drive native transduction channels by a photolysis of intracellulary loaded caged CAMP under the physiological condition. $V_{\mathrm{h}}=-50 \mathrm{mV}$. $\boldsymbol{E}$, Time dependence of the light-induced responses. Durations were 400, 800, 1200, 1600, 2000, 2400, and $2800 \mathrm{ms,} \mathrm{and} \mathrm{intensities} \mathrm{were} 0.62,0.31,0.21$, $0.15,0.12,0.10$, and 0.08 . $\boldsymbol{F}$, Current amplitudes in $\boldsymbol{E}$ were plotted against the duration of the light.

and Menini, 1997). Removal of $\mathrm{Ca}^{2+}$ feedback at positive potentials has been shown previously (Takeuchi and Kurahashi, 2003).

Figure $2 B$ shows current responses induced by the photolysis of caged cAMP under the absence of $\mathrm{Ca}^{2+}$ effects. In this experimental condition, the light-induced current was outward. Because the major ionic component included in the recording pipette was Cs ${ }^{+}$, and because $\mathrm{Cs}^{+}$has been shown to be permeable to the CNG channel (Kurahashi, 1990), it is highly likely that most of the outward current is carried by $\mathrm{Cs}^{+}$(Fig. 2A). The intensity-response relationship (Fig. $2 C$ ) could be fitted by the Hill equation with coefficient $\left(n_{H}\right)$ of $1.6 \pm 0.6(n=16)$, the value to be similar range as that obtained with inside-out patch preparations (1.2-1.6) (Nakamura and Gold, 1987; Kurahashi and Kaneko, 1991, 1993; Balasubramanian et al., 1996). Furthermore, $\mathrm{Ca}^{2+}$-dependent adaptational feature has become undetectable (data not shown) (but see Takeuchi and Kurahashi, 2003). Therefore, it is highly likely that the outward current induced by the photolysis of caged cAMP is carried through pure CNG channels. We could then fit the intensity-response curve with parameters that were obtained from previous experiments with inside-out patch preparations (Kurahashi and Kaneko, 1991) to specify absolute $[\text { cAMP }]_{i}$.

In the experiments, it was noticeable to see that the time course of current recovery after the termination of light was remarkably slower than that of the activation phase. This indicates that the cAMP removal (presumably by PDE and by the spatial diffusion) can be negligible for estimation of $[\mathrm{cAMP}]_{i}$ within this time window. This idea was further verified in the following experiments (Fig. 3).

Because it has been shown that CNG channels do not show remarkable desensitization (Kurahashi and Kaneko, 1993), the size of membrane current is simply assumed to reflect absolute $[\mathrm{cAMP}]_{\mathrm{i}}$ independent from the exposure time. However, some intrinsic natures and/or experimental manipulations may cause dose- and/or time-dependent changes in $[\mathrm{cAMP}]_{\mathrm{i}}$. To obtain AC activities in the following experiments, we first checked the time dependence of the $[\mathrm{cAMP}]_{\mathrm{i}}$ change with the UV exposure. One example to check such time dependence was to examine the reciprocity between the intensity and the duration of the light stimulation, as illustrated in Figure $3 B$. In this experiment, the cilia were illuminated with the UV light having different intensities, whereas the amount of light remained constant. Initially, the cell was stimulated by a brief and strong light illumination. Then, the light intensity was reduced to half with doubled exposure period. Similarly, the following stimuli were applied with weaker stimuli with longer exposures. This kind of experiment could specify how long the period the CAMP production expressed a liner function without receiving other timedependent factors. In such experiments, the peak amplitude was near constant as long as $2 \mathrm{~s}$ with little time dependence $(<10 \%$ reduction) (Fig. 3B). This result indicates that, in the period $<2 \mathrm{~s}, \Delta[\mathrm{cAMP}]_{\mathrm{i}}$ is simply determined by cAMP production.

We next obtained the relationship between $[\mathrm{cAMP}]_{\mathrm{i}}$ and appeared current size. In the experiment in Figure $4 A$, light steps were applied with different intensities. In this condition, $[\mathrm{cAMP}]_{\mathrm{i}}$ was thought to increase linearly as a function of time when the other factors were constant (Fig. 4B). [cAMP $]_{\mathrm{i}}(C)$ was then obtained from the membrane current with the inverse function of the Hill equation:

$$
C=\frac{K_{1 / 2}}{\left(\frac{I_{\max }}{I}-1\right)^{\frac{1}{n_{H}}}} .
$$

As predicted, $[\mathrm{cAMP}]_{\mathrm{i}}$ derived from the calculation increased monotonically with time (C-t curve) (Fig. $4 B$ ). Such monotonic increase in $[\mathrm{cAMP}]_{i}$ could be explained by an idea that $[\mathrm{cAMP}]_{\mathrm{i}}$ was increased within the ciliary cytoplasm during UV illumination.

There was, however, a slight deviation from the perfect linearity, and the curve generally showed a slight sublinear feature (Fig. $4 B)$. Because the light source of the UV system provides a constant intensity of light (see Materials and Methods), it is likely that this deviation occurs after the light is applied to the caged compounds. At this point, however, we could not identify whether this was attributable to the intrinsic feature of the olfactory receptor cell (e.g., nonlinear dose dependence of PDE) or attributable to the experimentations (e.g., depletion of the caged compound, some deterioration of the cell elements by constant UV application). If the reducing deviation was caused only by the depletion of the caged compound, the data interpretation for the following analyses would become very simple; we can ignore the apparent reduction observed here. It seems likely, however, that 

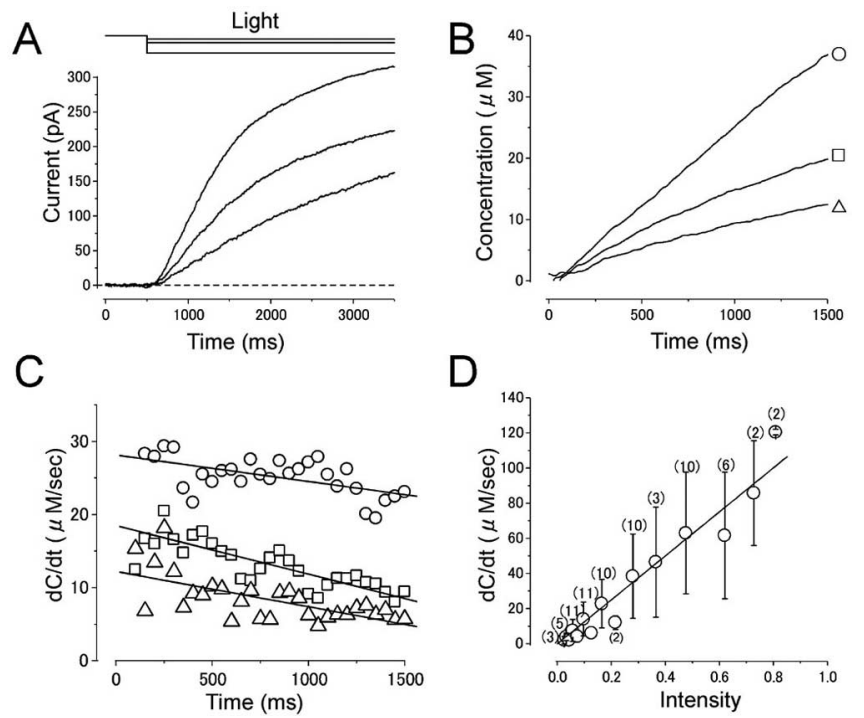

Figure 4. Absolute $[\mathrm{CAMP}]_{i}$ and $\mathrm{CAMP}$ production rate in the absence of $\mathrm{Ca}^{2+}$. $\boldsymbol{A}$, Current responses induced by different intensities of long light steps. Intensities were $0.03,0.06$, and 0.28 , while the duration was kept constant $(3 \mathrm{~s}) \cdot V_{\mathrm{h}}=+80 \mathrm{mV}$. The experimental condition is the same as in Figure $3 A$. B , Cytoplasmic CAMP concentration obtained by converting curves in $A$ with the inverse function of the Hill equation (Eq. 2). The parameters used were as follows: $n_{\mathrm{H}}$, $1.7 ; I_{\text {max }}, 176.5 \mathrm{pA} ; K_{1 / 2}, 22 \mu \mathrm{m}$. Time 0 corresponds to the initiation of stimuli. C, cAMP production rate obtained as a derivative of curve $\boldsymbol{B}$. Average slopes within $100 \mathrm{~ms}$ time windows was obtained from curve $\boldsymbol{B}$ at every $50 \mathrm{~ms}$. Symbols represent the same origins as those marked in $\boldsymbol{B}$. $\boldsymbol{D}$, Relationship between CAMP production rate and light intensity. The CAMP production rate was shown as a function of the light intensity. The straight line is the least-square fitting of the data points, which has a slope of 125 . Numbers in parentheses indicate the number of preparations. Note that the SD bar becomes larger as the light intensity is increased.

the reduction includes not only the depletion of caged cAMP, because the time course for deviation was not dependent on the light intensities (see below). This suggests that the slight deviation from the perfect linearity in the $C-t$ curve is caused, in part, by other reasons listed previously.

\section{Relationship between the light intensity and cAMP production rate}

Temporal kinetics of cAMP production rate (Fig. $4 C, r-t$ curve) was obtained by taking the derivative $(d C / d t)$ of Figure $4 B$. The cAMP production rate increased suddenly when the light step was applied. The amplitude of the rate change was dependent on the light intensity. During the constant light step, the cAMP production rate declined slightly and gradually. When the data points were fitted by a straight line, the average change in the production rate was $-21.2 \pm 11.6 \mu \mathrm{M} / \mathrm{s}^{2}$ (measured in four cells stimulated with a strong intensity). Again, it is highly likely that this reduction is caused by several reasons described before, and it is important to interpret following data taking this property into account.

In Figure $4 D$, cAMP production rates obtained at $r$ intercept (at $t=0$ ) from similar experiments done on 11 cells are plotted as a function of the light intensity. As predicted, the relationship was almost linear, providing rate $=125 \times$ intensity. SD bars became larger as the light intensity is increased. This seems to be a result of a variation of the responsiveness of cells to the light; individual cells show linear relationships between the production rate and light intensity. Because the photolysis of caged compound is unrelated to physiological conditions (including the membrane potential or presence/absence of $\mathrm{Ca}^{2+}$ ), the relationship shown in
Figure $4 D$ could be used as a standard curve throughout the present experiments.

\section{Light-induced current and $[\mathrm{CAMP}]_{\mathrm{i}}$ change in the physiological condition}

So far, the experiments were conducted in nonphysiological condition. We next re-examined light-induced responses under the physiological condition at the negative potential that has a normal concentration of $\mathrm{Ca}^{2+}$. Under the physiological condition, it is known that $\mathrm{Ca}^{2+}$ influx activates $\mathrm{Cl}^{-}$channels, which make high and nonlinear boosting of the total membrane current. Furthermore, it has been shown that $\mathrm{Ca}^{2+}$ influx causes a negative feedback to the olfactory transduction system to express olfactory adaptation (Kurahashi and Shibuya, 1990; Zufall et al., 1991; Kurahashi and Menini, 1997), which is actually regulated by the reduction of cAMP affinity of the CNG channel (Chen and Yau, 1994; Shapiro and Zagotta, 2000; Bradley et al., 2001). These $\mathrm{Ca}^{2+}$ effects would cause changes in the relationship between the membrane current and $[\mathrm{cAMP}]_{\mathrm{i}}$, which was needed to be reexamined with reference to the parameters obtained previously in the absence of $\mathrm{Ca}^{2+}$ effects.

First, the reciprocity between the intensity and duration in the light-induced response was re-examined with the same protocol as that used in the absence of $\mathrm{Ca}^{2+}$. In the physiological condition, the time-dependent decline became more obvious than that observed in $\mathrm{Ca}^{2+}$-free conditions, especially at exposures longer than $\sim 1 \mathrm{~s}$ (Fig. $3 E, F$ ). This observation is reasonable, because in this condition, $\mathrm{Ca}^{2+}$-dependent adaptation starts to work. Furthermore, it has been reported that $\mathrm{Ca}^{2+}$-activated $\mathrm{Cl}^{-}$channel, as a major pathway carrying the olfactory transduction current, shows a slight desensitization (Reisert et al., 2003). Average time for $10 \%$ reduction of the current was $1.2 \mathrm{~s}$ (SD $0.5 ; n=9)$.

Next, different intensities of light steps were applied to induce membrane currents to obtain $[\mathrm{cAMP}]_{\mathrm{i}}$ as a function of time. Under the physiological condition, however, $[\mathrm{CAMP}]_{\mathrm{i}}$ could not be directly estimated because of an addition of $\mathrm{Ca}^{2+}$-related cascades; $n_{H}$ might have been different and $K_{1 / 2}$ was unknown. Furthermore, it was reasonably considered that the time dependence of the system was different from that observed at the $\mathrm{Ca}^{2+}$-free condition. Therefore, we used the following steps to estimate $[\mathrm{cAMP}]_{\mathrm{i}}$ and its time dependence within the cilia. First, the intensity-response relationship of light-induced responses was obtained to specify $n_{H}$ (5.1) (Takeuchi and Kurahashi, 2002). Second, light steps with different intensities were applied to obtain the light-induced current as a function of time (Fig. 5A). Third, the current traces were converted with a formula (Eq. 2) to the $C-t$ curve (Fig. $5 B$ ) remaining $K_{1 / 2}$ as an unknown constant. Fourth, $r-t$ curve (Fig. $5 C$ ) was obtained as a derivative of $C-t$ curve including $K_{1 / 2}$ still as an unknown constant. The resultant $r-t$ curve expressed apparent reduction as observed previously. Here, $r$ intercept (therefore at $t=0$ ) must be identical to the cAMP production rate that was obtained under the $\mathrm{Ca}^{2+}$-free condition at identical light intensity (therefore, the slope of the curve is 125) (Fig. 5D). $K_{1 / 2}$ was then obtained from the leastsquare fitting of the data points, being $32.9 \pm 16.8 \mu \mathrm{M}(n=9)$. The $[\mathrm{cAMP}]_{\mathrm{i}}$ illustrated in Figure $5, B$ and $C$, was determined after $K_{1 / 2}$ was obtained through these procedures.

The resultant $C-t$ curve expressed a monotonic increase during the constant UV step. This represents that the $[\mathrm{cAMP}]_{\mathrm{i}}$ increases gradually, because they are produced constantly by the photolysis of caged cAMP. The slight deviation from the perfect first-order function was observed as in the $\mathrm{Ca}^{2+}$-free condition. Again, it is highly likely that this deviation is a result of some 

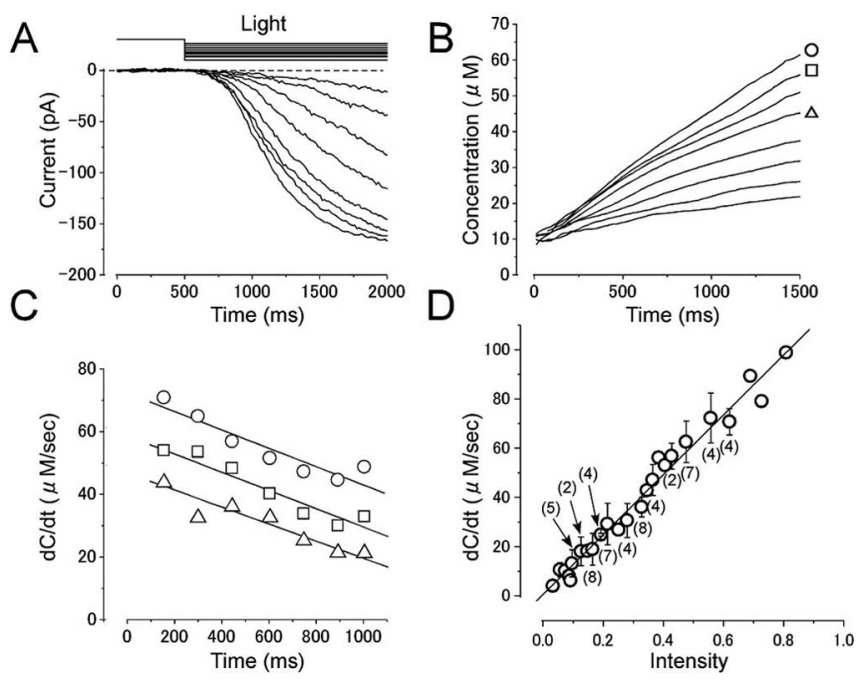

Figure 5. cAMP production rate under the physiological condition (same condition as in Fig. 3D). $A$, Current responses induced by different intensity of the light steps. Intensities: $0.09,0.13$, $0.16,0.21,0.28,0.33,0.37$, and $0.48 . V_{\mathrm{h}}=-50 \mathrm{mV}$. B, Cytoplasmic cAMP concentration. Curves were obtained by converting curves in $A$ with the inverse function of the Hill equation (Eq. 2). Absolute concentration was determined after we obtained $K_{1 / 2}(33 \mu \mathrm{m})$ at $\boldsymbol{D}$ (see Results). Time 0 corresponds to the initiation of stimuli. Symbols represent data used for the illustration in C. C, cAMP production rate obtained as a derivative of the curve $\boldsymbol{B}$. Intensities: $0.28,0.37$, and 0.48 . Average slopes within $320 \mathrm{~ms}$ time windows were obtained from curves in $\boldsymbol{B}$ for every $150 \mathrm{~ms}$. D, Relationship between cAMP production rate and light intensity. The straight line has a slope of 125 .

mechanisms that influence to some points after the light is applied to caged compound. Because of this, the resultant $r-t$ curve expressed declining feature. The shape could be fitted by a near negative-linear function. The reduction rate was $66.2 \pm 63.2$ $\mu \mathrm{M} / \mathrm{s}^{2}(n=4)$, which was not statistically different from that obtained in the absence of $\mathrm{Ca}^{2+}$. This may be explained by a fact that the time dependence of cAMP-induced current becomes obvious beyond $1 \mathrm{~s}$. Again, we emphasize that this apparent reduction is not attributable to the real reduction in the cAMP production rate.

In 2 of 11 cells, the intensity-response relationship was not described simply by the single Hill equation, presumably because of an appearance of the pure CNG component at small responses [Takeuchi and Kurahashi (2002), their Fig. 8]. Such cells showed biphasic or complicated $r-t$ curve when examined with the same protocol. At this point, unfortunately, we do not know the precise reason why pure $\mathrm{CNG}$ component becomes obvious in those cells. In the present study, these cells were not used for analysis to simplify the logics. Detailed analyses will be made elsewhere.

\section{$[\mathrm{cAMP}]_{\mathrm{i}}$ change and adenylyl cyclase activity during the ligand application}

$[\mathrm{cAMP}]_{\mathrm{i}}$ change and $\mathrm{AC}$ activities induced by the odorant were estimated (Fig. 6) by using the parameters obtained with UV light experiments at the physiological condition (Fig. 5). When the odorant stimulation was applied from the puffer pipette as a long step with different pressures, the current started to develop gradually $\sim 200 \mathrm{~ms}$ after the onset of the stimulus (Fig. 6B). Obviously, the time lag was attributable to a delayed increase of $[\mathrm{cAMP}]_{\mathrm{i}}$ (Lowe and Gold, 1993a; Takeuchi and Kurahashi, 2002). The current-time curve was then converted to represent $[\mathrm{cAMP}]_{\mathrm{i}}$ as a function of time ( $C-t$ curve) by a formula (Eq. 2$)$ with parameters obtained with light stimulation of this cell. Essentially, $[\mathrm{cAMP}]_{\mathrm{i}}$ started to increase a few hundred milliseconds
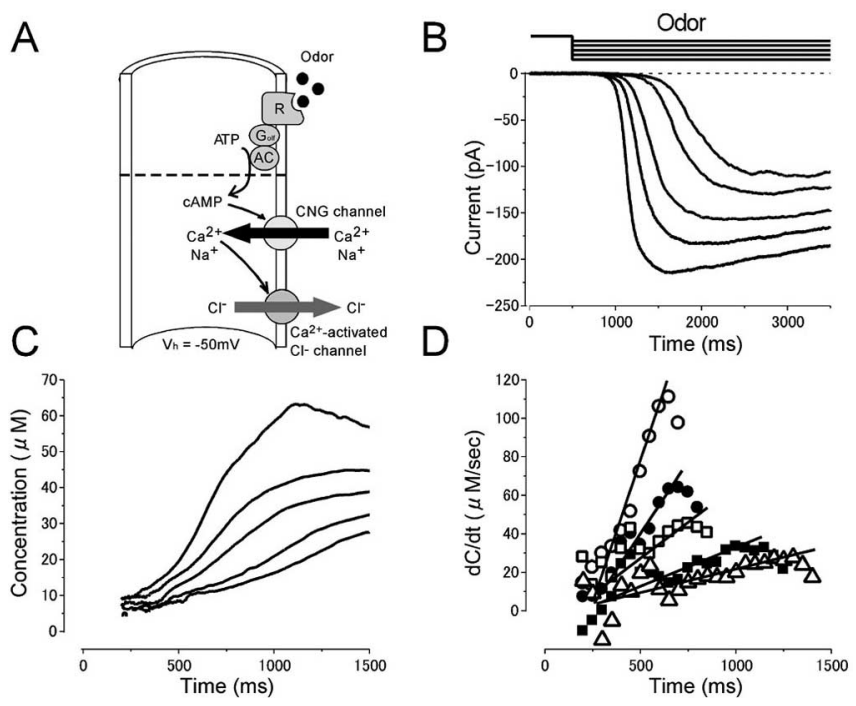

Figure 6. Odor-induced current, $[\mathrm{CAMP}]_{\mathrm{i}}$ change, and CAMP production rate during ligand application under the physiological condition. $\boldsymbol{A}$, Experimental logic. Experiments were conducted to drive native transduction pathway under the physiological condition. $V_{\mathrm{h}}=-50 \mathrm{mV}$. $\boldsymbol{B}$, Current responses induced by different intensity of the long odor steps $(6.5,7,10,12$, and 15 $\mathrm{kPa}$ ) while the duration was kept constant (3 s). C, Cytoplasmic CAMP concentration. Curves were obtained by converting curves in $\boldsymbol{B}$ with the inverse function of the Hill equation (Eq. 2). $I_{\max }$ obtained by light stimulation of this cell was $223 \mathrm{pA} . K_{1 / 2}(33 \mu \mathrm{m})$ was obtained from the experiment in Figure 5. Time 0 corresponds to the initiation of stimuli. Because in our protocol the C value cannot be obtained when the original membrane current is zero, the data conversion was conducted after the current started to develop ( $200 \mathrm{~ms}$ after the onset of the stimuli). D, CAMP production rate obtained as a derivative of curve $\boldsymbol{C}$. Average slopes within $100 \mathrm{~ms}$ time windows were obtained from curves in $C$ for every $50 \mathrm{~ms}$. The straight lines were drawn by the least-square fitting. As in C, CAMP production rates were plotted $200 \mathrm{~ms}$ after the onset of the stimuli. The rising phase was fitted by the straight line with the least-square method.

after the onset of the stimulus and went on developing as time processed in the time range up to a second (Fig. 6C). The maximum $[\mathrm{cAMP}]_{\mathrm{i}}$ was $61 \mu \mathrm{M}$ in the cell illustrated in Figure 6 . The maximum $[\mathrm{cAMP}]_{\mathrm{i}}$ observed among four cells tested in the present study was $129 \mu \mathrm{M}$.

The rising phase of the $C-t$ curve was obviously superlinear. This is an interesting point, because, with UV light experiments in which the cAMP production rate is constant, the $C-t$ curve was almost linear or even sublinear. By comparing two $C-t$ curves (Figs. $5 B, 6 C$ ), it is assumed that the cAMP production rate increases as the time processed with odor stimulation. By taking the derivative, cAMP production rates that represent odorantactivated AC activities were obtained and plotted as a function of time (Fig. 6D). After a silent period of a few hundred milliseconds, AC activities started to increase with an almost linear function with time. The slope of the rising phase became steeper as the concentration of the odorant was increased. Obviously, the absolute number of AC molecules activated by the odorant is dependent on the stimulus concentration. After the peak value, AC activity gradually decreased. The highest AC activity differed from cell to cell. This feature can be explained by the fact that individual olfactory cells express different receptor proteins having different affinities to odorants. The average of maximum AC activities was $96 \mu \mathrm{M} / \mathrm{s}(n=4)$.

It is likely that this $r-t$ curve has already been affected by the time dependence of the olfactory system as described before. Essentially, however, the conclusion for the monotonic increase of $\mathrm{AC}$ activity would not be affected by the time dependence of the olfactory system, because the $r-t$ curve in the photolysis experiment showed a reduction; vice versa, the $r-t$ curve in the odorant 
experiments was a monotonic increase. One may even think that the $r-t$ curves obtained in odor responses could simply be converted into a second-order function by the reduction rate of the $r-t$ curves obtained in the light experiments. Without knowing the precise mechanisms, however, simple compensation may lead to an inappropriate data manipulation. Rather, the values obtained here would be compensated after we understand the entire molecular mechanisms.

With the AC activity and the ciliary volume, we calculated the number of cAMP molecules produced in the cilia at the maximum response. The diameter of olfactory cilia has been reported to be $0.2 \mu \mathrm{m}$ and the length $10 \mu \mathrm{m}$ (Menco, 1980). Single newt olfactory receptor cells have $\sim 10$ cilia on average. Such morphological shape and AC activity obtained here gave 180,000 molecules/s/cell. Considering the experimental processes and significant figure, we conclude that single olfactory receptor cell produced cAMP in an order of $2 \times 10^{5}$ molecules/s/cell at the maximum. One may pay attention to the fact that PDE activities are enhanced by $\mathrm{Ca}^{2+}$ influx (Anholt and Rivers, 1990; Borisy et al., 1992). However, note that our conclusion for the AC activities is not influenced by the $\mathrm{Ca}^{2+}$ dependence of PDE activity, because cAMP production is unrelated from other physiological factors. However, the actual $[\mathrm{cAMP}]_{\mathrm{i}}$ value obtained in the present experimental protocols may be an overestimation, if we take the $\mathrm{Ca}^{2+}$ effect on PDE into account. Even for this, our present protocol of measuring $[\mathrm{cAMP}]_{\mathrm{i}}$ at an early time stage of responses at which little $\mathrm{Ca}^{2+}$ influx is expected must have reduced the experimental error.

\section{Discussion}

In the present study, we estimated temporal kinetics and activities for both AC and cytoplasmic cAMP. It was shown that $[\mathrm{cAMP}]_{\mathrm{i}}$ increased superlinearly with time for $>1 \mathrm{~s}$ during the odorant stimulation, which was because of a gradual increase of cAMP production rate. So far, there was very limited information about the molecular activities of olfactory transduction elements, whereas G-protein-mediated signal transduction has been quite well documented in works done on the rod photoreceptor cell (Pugh and Lamb, 1990; Lamb and Pugh, 1992). In the present study, we overcame difficulties accompanied with the properties (heterogeneous responsiveness) and structure (fine cilia) of the olfactory receptor cell. One may concern about the hydrolysis and diffusion of cAMP during the present work. As long as the activities of both are constant, data interpretation and values obtained here would provide reasonable conclusions. Also, note that our estimations were performed in the time window in which time dependence did not work so drastically (Fig. 3).

In previous studies, the temporal change of [cAMP] was measured with the biochemical assay applied to detached ciliary preparations (and therefore open preparations) (Breer et al., 1990; Boekhoff and Breer, 1992; Restrepo et al., 1993). Rapid kinetics of [cAMP] change was measured especially with so-called the "rapid quench method." In those experiments, [cAMP] change was very transient with a peak value of $\sim 80 \mathrm{~ms}$ after the onset of odorant stimulation (Breer et al., 1990). For a long period of time, such a transient time course in [cAMP] change has provided the question of why odorant responses in single receptor cells last for a couple of seconds even with a very brief pulse of stimulation (e.g., $50 \mathrm{~ms}$ ). The present study showed, however, that $[\mathrm{cAMP}]_{\mathrm{i}}$ increases superlinearly with time for $>1 \mathrm{~s}$ and thus resolved a longstanding question. At this point, we do not know what the main cause of the data discrepancy is, and there are actually varieties of possibilities. Among them, the most intriguing possibility is the presence of cytoplasmic soluble factors that maintain the G-protein-mediated enzymatic cascade. Such factors would be washed out if one brings the preparation into in vitro condition. Especially, olfactory transduction takes place at the fine cilia that have a very small internal volume. Cytoplasmic factors would be almost completely diluted by the surrounding media. It would be worth trying to survey such factors in the olfactory receptor cell.

The observation that the effecter enzyme shows monotonic increase during the ligand stimulation is essentially consistent with that estimated on rod photoreceptor cells (Pugh and Lamb, 1990; Lamb and Pugh, 1992; Pugh et al., 1999), which have a similar transduction system as the olfactory system in terms of the G-protein-mediated signaling cascade. In the model used for the rod photoreceptor cell, rhodopsin activation was set to be constant during the course of the PDE activation. In the present study, we applied odorant stimulation with steps and obtained essentially the same result as in the rod in terms of the temporal kinetics of the effecter enzyme activity. This may give us some hints to consider about the receptor-ligand interaction. However, at this stage, the time course of the AC activation is not yet well established, especially at the point of whether it is linear. For the ligand-receptor interaction, it would be important to investigate directly the lifetime of single odor receptor protein and ligand binding/dissociation processes.

In the photoreceptor cell, it is widely known that even a single photoisomelization leads to the change in cGMP by 250,000 molecules (for review, see Stryer, 1986), and it is thought to be a molecular basis of signal amplification of the rod. In comparison, the present study showed that the olfactory receptor cell had extremely low signal amplification mechanisms on their enzymatic processes ( $\sim 200,000$ molecules/s/cell at the maximum). Even if there are some experimental errors in these numbers, the differences are anomalously large. In fact, furthermore, in the olfactory receptor cell, the least effective dose for the odorant stimulation is a range of micromolar (Araneda et al., 2000) and a saturating dose of $100 \mu \mathrm{M}$ to $1 \mathrm{~mm}$. This concentration range is almost equivalent to that of $[\mathrm{cAMP}]_{\mathrm{i}}$ during the odorant responses (Fig. 6). This also supports the notion that there is a little molar amplification in the olfactory enzymatic cascade. Seemingly, such low amplification is thought to be a disadvantage for the signal transduction. In contrast, however, one would notice a big difference between the olfactory and photoreceptor cells; cAMP is produced by odor in olfaction, whereas cGMP is hydrolyzed by light in vision. In the rod, basal $[\mathrm{cGMP}]_{\mathrm{i}}$ is already determined by some homeostatic regulations to a constant level that is independent on the light stimulation. In contrast, in olfaction, odorant activation of GPCR leads to the production of cAMP with the number determined by the amplification factor multiplied by the number of GPCR molecules. If single olfactory GPCR activation produced a large number of second messenger molecules as the rod, a fully liganded condition might produce a large amount of cAMP molecules, which is equivalent to the hydrolysis of a large number of ATP molecules. ATP is used for activities of cells in many aspects (maintenance of electrochemical gradient by $\mathrm{Na}^{+} / \mathrm{K}^{+}$ATPase, cell movements, phosphorylation, etc.). In addition, cilia do not have mitochondria, and therefore ATP must be supplied from the olfactory knob. Diffusion of ATP along the ciliary lumen would limit the ATP concentration within the cilia. Therefore, the low amplification at the level of enzymes in the olfactory cilia would be established to express an efficient energy conversion using a small number of second-messenger molecules.

Apparently, signal transduction with a smaller number of 
second-messenger formation in the olfactory receptor cell could be achieved by the fine ciliary structure that has a high surfacevolume ratio. In addition, the olfactory receptor cell might have acquired a very nonlinear amplification (Fig. 1) at downstream points from the cAMP generation site, by the sequential openings of CNG channels and $\mathrm{Ca}^{2+}$-activated $\mathrm{Cl}^{-}$channels (Kurahashi and Yau, 1993, 1994; Lowe and Gold, 1993b; Takeuchi and Kurahashi, 2002), using $\mathrm{Ca}^{2+}$ as a third messenger, which is not present in the phototransduction system.

\section{References}

Anholt RRH, Rivers AM (1990) Olfactory transduction: cross-talk between second-messenger systems. Biochemistry 29:4049-4054.

Araneda RC, Kini AD, Firestein S (2000) The molecular receptive range of an odorant receptor. Nat Neurosci 12:1248-1255.

Bakalyar HA, Reed RR (1990) Identification of a specialized adenylyl cyclase that may mediate odorant detection. Science 250:1403-1406.

Balasubramanian S, Lynch JW, Barry PH (1996) Calcium-dependent modulation of the agonist affinity of the mammalian olfactory cyclic nucleotide-gated channel by calmodulin and a novel endogenous factor. J Memb 152:13-23.

Boekhoff H, Breer I (1992) Second messenger signaling in olfaction. Curr Opin Neurobiol 2:439-443.

Borisy FF, Ronett GV, Cunningham AM, Juilfs D, Beavo J, Snyder SH (1992) Calcium/calmodulin-activated phosphodiesterase expressed in olfactory receptor neurons. J Neurosci 12:915-923.

Bradley J, Reuter D, Frings S (2001) Facilitation of calmodulin-mediated odor adaptation by cAMP-gated channel subunits. Science 294:2176-2178.

Breer H, Boekhoff I, Tareilus E (1990) Rapid kinetics of second messenger formation in olfactory transduction. Nature 345:65-68.

Buck L, Axel R (1991) A novel multigene family may encode odorant receptors: a molecular basis for odor recognition. Cell 65:175-187.

Chen TY, Yau KW (1994) Direct modulation by $\mathrm{Ca}^{2+}$-calmodulin of cyclic nucleotide-activated channel of rat olfactory receptor neurons. Nature 368:545-548.

Firestein S, Shepherd GM, Werblin FS (1990) Time course of the membrane current underlying sensory transduction in salamander olfactory receptor neurons. J Physiol (Lond) 430:135-158.

Hamill OP, Marty A, Neher E, Sakmann B, Sigworth FJ (1981) Improved patch-clamp techniques for high-resolution current recording from cells and cell-free membrane patches. Plfügers Arch 391:85-100.

Ito Y, Kurahashi T, Kaneko A (1995) Pressure control instrumentation for drug stimulation. Nippon Seirigaku Zasshi 57:127-133.

Jones DT, Reed RR (1989) Golf: an olfactory neuron specific-G protein involved in odorant signal transduction. Science 244:790-795.

Kleene SJ (1997) High-gain, low-noise amplification in olfactory transduction. Biophys J 73:1110-1117.

Kleene SJ, Gesteland RC (1991) Calcium-activated chloride conductance in frog olfactory cilia. J Neurosci 11:3624-3629.

Kurahashi T (1989) Activation by odorants of cation-selective conductance in the olfactory receptor cell isolated from the newt. J Physiol (Lond) 419:177-192.

Kurahashi T (1990) The response induced by intracellular cyclic AMP in isolated olfactory receptor cells of the newt. J Physiol (Lond) 430:355-371.

Kurahashi T, Kaneko A (1991) High density cAMP-gated channels at the ciliary membrane in the olfactory receptor cell. NeuroReport 2:5-8.

Kurahashi T, Kaneko A (1993) Gating properties of the cAMP-gated channel in toad olfactory receptor cells. J Physiol (Lond) 466:287-302.
Kurahashi T, Menini A (1997) Mechanism of odorant adaptation in the olfactory receptor cell. Nature 385:725-729.

Kurahashi T, Shibuya T (1990) $\mathrm{Ca}^{2+}$-dependent adaptive properties in the solitary olfactory receptor cell of the newt. Brain Res 515:261-268.

Kurahashi T, Yau KW (1993) Co-existence of cationic and chloride components in odorant-induced current of vertebrate olfactory receptor cells. Nature 363:71-74.

Kurahashi T, Yau KW (1994) Olfactory transduction. Tale of an unusual chloride current. Curr Biol 4:256-258.

Lamb TD, Pugh Jr EN (1992) A quantitative account of the activation steps involved in phototransduction in amphibian photoreceptors. J Physiol (Lond) 449:719-758.

Lowe G, Gold GH (1991) The spatial distributions of odorant sensitivity and odorant-induced currents in salamander olfactory receptor cells. J Physiol (Lond) 442:147-168.

Lowe G, Gold GH (1993a) Contribution of the ciliary cyclic nucleotidegated conductance to olfactory transduction in the salamander. J Physiol (Lond) 462:175-196.

Lowe G, Gold GH (1993b) Nonlinear amplification by calcium-dependent chloride channels in olfactory receptor cells. Nature 366:283-286.

Lowe G, Nakamura T, Gold GH (1989) Adenylate cyclase mediates olfactory transduction for a wide variety of odorants. Proc Natl Acad Sci USA 86:5641-5645.

Matthews HR, Reisert J (2003) Calcium, the two-faced messenger of olfactory transduction and adaptation. Curr Opin Neurobiol 13:469-475.

Menco BP (1980) Qualitative and quantitative freeze-fracture studies on olfactory and nasal respiratory structures of frog, ox, rat, and dog. I. A general survey. Cell Tissue Res 207:183-209.

Nakamura T, Gold GH (1987) A cyclic nucleotide-gated conductance in olfactory receptor cilia. Nature 325:442-444.

Pace U, Hanski E, Salomon Y, Lancet D (1985) Odorant-sensitive adenylate cyclase may mediate olfactory reception. Nature 316:225-258.

Pugh Jr EN, Lamb TD (1990) Cyclic GMP and calcium: the internal messengers of excitation and adaptation in vertebrate photoreceptors. Vision Res 30:1923-1948.

Pugh Jr EN, Nikonov S, Lamb TD (1999) Molecular mechanisms of vertebrate photoreceptor light adaptation. Curr Opin Neurobiol 4:410-418.

Reisert J, Bauer PJ, Yau KW, Frings S (2003) The Ca-activated Cl channel and its control in rat olfactory receptor neurons. J Gen Physiol 122:349-363.

Restrepo D, Boekhoff I, Breer H (1993) Rapid kinetic measurements of second messenger formation in olfactory cilia from channel catfish. Am J Physiol 264:906-911.

Shapiro MS, Zagotta WN (2000) Structural basis for ligand selectivity of heteromeric olfactory cyclic nucleotide-gated channels. Biophys J 78:2307-2320.

Sklar PB, Anholt RRH, Snyder SH (1986) The odorant-sensitive adenylate cyclase of olfactory receptor cells. Differential stimulation by distinct classes of odorants. J Biol Chem 261:15538-15543.

Stryer L (1986) Cyclic GMP cascade of vision. Annu Rev Neurosci 9:87-119.

Takeuchi H, Kurahashi T (2002) Photolysis of caged cyclic AMP in the ciliary cytoplasm of the newt olfactory receptor cell. J Physiol (Lond) 541:825-833.

Takeuchi H, Kurahashi T (2003) Identification of second messenger mediating signal transduction in the olfactory receptor cell. J Gen Physiol 122:557-567.

Takeuchi H, Imanaka Y, Hirono J, Kurahashi T (2003) Cross-adaptation between olfactory responses induced by two subgroups of odorant molecules. J Gen Physiol 122:255-264.

Zufall F, Shepherd GM, Firestein S (1991) Inhibition of the olfactory cyclic nucleotide gated ion channel by intracellular calcium. Proc R Soc Lond B Biol Sci 246:225-230. 